\title{
NuklearMedizin 2019 in Bremen
}

Vom 3. bis 6. April 2019 wird die die 57. Jahrestagung der Deutschen Gesellschaft für Nuklearmedizin auf dem Gelände der MESSE BREMEN stattfinden.

Der Kongresspräsident, Herr Professor Michael Schäfers (Münster), sowie der Vorsitzende des wissenschaftlichen Komitees, Herr Professor Markus Essler (Bonn), freuen sich auf eine lebendige Tagung und einen regen fachlichen Austausch.

In diesem Jahr erwarten Sie bei der Jahrestagung der DGN spannende Neuerungen:

So werden Referenten in Leuchtturm-Sitzungen richtungsweisende Entwicklungen in den Disziplinen der Nuklearmedizin präsentieren - dies in Form eines einführen- den Übersichtsvortrags sowie ausgewählter Abstracts.

Zudem werden im Zukunftsworkshop am Samstag, 6. April 2019, „Zündfunken“ für die Nuklearmedizin der Zukunft gesetzt. Mit Impulsvorträgen renommierter Vertreter unseres Faches, unserer Nachbarfächer, der Industrie und der Kostenträger geht es um die Thematik, wieviel Nuklearmedizin unsere Zukunft braucht.

Zusätzlich wird dem wissenschaftlichen Nachwuchs auf der NuklearMedizin 2019 mit speziellen Formaten Raum für eigenständige Programme geboten.

Die Wolfgang Becker Gedächtnisvorlesung hält in diesem Jahr Herr Professor Kurt
Werner Schmid vom Institut für Pathologie des Uniklinikums Essen, der im Rahmen unserer Jahrestagung mit der korrespondierenden Mitgliedschaft der DGN geehrt wird.

Das vielseitige wissenschaftliche Programm des Kongresses wird durch ein Fortbildungsprogramm ergänzt, welches das erste Modul des Fortbildungs-Curriculums 2019-2021 umfasst. Dieses wurde vom DGN-Ausschuss Fort- und Weiterbildung unter dem Vorsitz von Herrn Professor Winfried Brenner erarbeitet.

Wegen der großen Resonanz in den Vorjahren wird es auf der Jahrestagung sowohl einen Kodierworkshop als auch wieder zwei Nuklearmedizin- und Radiologie-Foren geben. Die Foren sind gemeinsam von der Deut- 
schen Gesellschaft für Nuklearmedizin (DGN) und der Deutschen Röntgengesellschaft (DRG) unter Federführung der Interdisziplinären Arbeitsgemeinschaft für hybride Bildgebung (ID AG Hybride BG) organisiert. Vertreter beider Fachgesellschaften referieren hier zu ihren jeweiligen Schwerpunkten.

Gerade ist das S3-Leitlinien-Projekt Schilddrüsenkarzinom unter der Federführung der DGN, DGE und der Chirurgischen Arbeitsgemeinschaft Endokrinologie der DGAV gestartet. Passend dazu findet zum Auftakt der Jahrestagung am Mittwoch, 3. April
2019, das Vorkongress-Symposium „Therapie des Differenzierten Schilddrüsenkarzinoms: Aktuelle Leitlinien - aktuelle Kontroversen" statt. Die wissenschaftliche Leitung des Vorkongress-Symposiums liegt bei Professor Markus Essler.

Es werden neben erfahrenen Nuklearmedizinern auch Referenten aus den anderen Fächern, die an der Behandlung des differenzierten Schilddüsenkarzinoms beteiligt sind (Endokrinologie, Onkologie, Chirurgie, Pathologie) an dem Vorkongress-Symposium teilnehmen, um dieses wichtige Thema interdisziplinär und auch kontrovers zu diskutieren.
Noch bis zum 25. März 2019 können Sie sich auf der Homepage nukmed19.nuklearmedizin.de unter der Rubrik „Teilnahme“ als Teilnehmer zur Jahrestagung und auch zum Vorkongress-Symposium registrieren. Auf der Kongresshomepage finden Sie zudem das aktuelle Programm sowie weitere Informationen zur Jahrestagung.

Wir freuen uns auf ein Wiedersehen zur NuklearMedizin 2019 in Bremen! 